\title{
Trends in Incident and Recurrent Rates of First-Ever Ischemic Stroke in Taiwan between 2000 and 2011
}

\author{
Meng Lee, ${ }^{a}$ Yi-Ling Wu, ${ }^{a}$ Bruce Ovbiagele ${ }^{\mathrm{b}}$ \\ ${ }^{a}$ Department of Neurology, Chang Gung University College of Medicine, Chang Gung Memorial Hospital, Chiayi branch, Taiwan \\ ${ }^{b}$ Department of Neurosciences, Medical University of South Carolina, Charleston, South Carolina, USA
}

Background and Purpose The burden of stroke is comparatively greater in Asian countries than in the Western world. While there has been a documented recent decline in the incidence of stroke in several Western nations due to better risk factor management, much less is known about the nature and trajectory of stroke in Asia over the last decade. The objective of this study was to explore risk factors, medication use, incidence, and one-year recurrence of stroke in Taiwan.

Methods We conducted a nationwide cohort study by reviewing all hospitalized patients ( $\geq$ 18 years) with a primary diagnosis of ischemic stroke between 2001 and 2011 from Taiwan National Health Insurance Research Database.

Results A total of 291,381 first-ever ischemic stroke patients were enrolled between 2000 and 2011. The average age was about 70 years and approximately $58.6 \%$ of them were men. While prevalence of diabetes mellitus and hyperlipidemia, as well as use of statins, antiplatelet agents, and oral anticoagulant agents for atrial fibrillation significantly increased; incidence (142.3 vs. 129.5 per 100,000 in 2000 and 2011, respectively) and one-year recurrence ( $9.6 \%$ vs. $7.8 \%$ in 2000 and 2011, respectively) of stroke declined during this period of time. Conclusions Over the last decade in Taiwan, rates of primary ischemic stroke and one-year recurrent stroke decreased by $9 \%$ and $18 \%$ respectively.

Keywords Ischemic stroke; Incidence; Recurrence; Cohort

\author{
Correspondence: Meng Lee \\ Department of Neurology, Chang Gung \\ University College of Medicine, \\ Chang Gung Memorial Hospital, \\ Chiayi branch, 6 West Section, \\ Chiapu Road, Puzi, Taiwan \\ Email: bluccc@adm.cgmh.org.tw
}

Received: July 20, 2015 Revised: October 23, 2015 Accepted: October 25, 2015

The authors have no financial conflicts of interest.

\section{Introduction}

Compared with a nonfatal myocardial infarction, a nonfatal stroke causes a 1.5-fold greater loss of disability-adjusted lifeyear. ${ }^{1}$ Nonetheless, over the past decade there has been a clear and impressive decline in the incidence of stroke in several Western nations. ${ }^{2-5}$ The positive change in the occurrence of stroke in these countries has been attributed primarily to better screening and treatment of established risk factors for stroke, particularly hypertension. ${ }^{6}$ The burden of stroke is greater in Asian countries than in the Western world, including a substantially higher incidence of stroke than myocardial infarc- tion. ${ }^{7-9}$ However, data on the extent to which several Asia countries have recently fared with regard to improvement in stroke outcomes are relatively sparse. One study suggested the incidence of stroke decreased from 1961 to 2000 in Japan $^{7}$ and an overview study revealed that recurrent stroke decreased in each decade from 1960 to $2010 .{ }^{10}$ Certainly more nationwide data on the evolving nature and trajectory of stroke in Asia are needed for research and planning purposes. The objective of this study was to explore risk factors, medication use, incidence, and one-year recurrence of stroke using the entire population in Taiwan by National Health Insurance Research Database (NHIRD). 


\section{Methods}

\section{Study design and dataset}

We retrospectively assessed data collected 2000 through 2012, from Taiwan NHIRD. Taiwan has implemented a singlepayer, compulsory National Health Insurance program in 1995, which includes reimburses of outpatient visits, hospital admissions, prescriptions for $99 \%$ of Taiwanese population. All contracted institutions must file claims according to standard formats, which are subsequently transformed into NHIRD.

\section{Study population}

We identified all hospitalized patients ( $\geq 18$ years) who were admitted with a primary diagnosis of ischemic stroke (International Classification of Diseases, Ninth Revision codes 433, 434, 436) for the first time between 2000 and 2011. A prior study validating the diagnosis of acute ischemic stroke in National Health Insurance claims data using the Taiwan Stroke Registry as a reference revealed a positive predictive value of $88.4 \%$ and sensitivity of $97.3 \% .{ }^{11}$ Another cross-sectional study showed that among confirmed cases of acute ischemic stroke in a hospital medical record, 94.5\% were assigned 'ischemic stroke' as the principal diagnosis in the NHIRD. ${ }^{12}$ We only enrolled patients receiving brain computed tomography (CT) or magnetic resonance imaging (MRI) during hospitalization with the assumption that all patients with symptoms of acute stroke should receive brain imaging. This approach was intended to exclude stroke patients who were hospitalized for rehabilitation during chronic stage. Although brain imaging, especially $\mathrm{CT}$, may not be able to reveal the cerebral infarction in patients with symptoms or signs of acute stroke, it can easily exclude intracranial hemorrhage. Thus NHIRD had a low probability of misclassifying hemorrhagic stroke into ischemic stroke if brain imaging was done.

The first-ever ischemic stroke during study period was defined as the index stroke. Patients were excluded if they had history of any strokes between 1997 and 1999. Patients were classified into three groups according to a year of stroke occurrence, which are 2000-2003, 2004-2007, and 2008-2011. This was a nationwide study that included all available and eligible patients.

\section{Main outcome measures}

The primary outcome of this study was incidence of ischemic stroke in Taiwan. The incidence rate was defined as numbers of first-ever stroke patients each year divided by midyear population per 100,000 population. The leading secondary outcome was the one-year stroke recurrent rate, defined as numbers of patients with first recurrent stroke (ischemic or hemorrhagic) within one year after index stroke divided by numbers of index stroke patients. The followed-up started from index stroke to stroke recurrence, or one year after index stroke, whichever came first.

\section{Statistical analysis}

Baseline characteristics were presented as medians (interquartile ranges) for continuous variables and percentages for categorical variables, as appropriate. All statistics analyses were performed with SAS statistical software, version 9.2 (SAS Institute Inc., Cary, USA).

\section{Results}

A total of 291,381 first-ever ischemic stroke patients were enrolled between 2000 and 2011. The average age was about 70 years and approximately $58.6 \%$ of them were men. The average length of hospital stay was about 7 days. More than half of patients had history of hypertension, which was from 59\% to 63\% and the frequency of hypertension in ischemic stroke was not much different in three time period. The frequencies of diabetes mellitus (from $29.6 \%$ to $34.0 \%$ ) and hyperlipidemia (from $22.0 \%$ to $40.6 \%$ ) increased steadily during study periods. Frequencies of atrial fibrillation (from $7.3 \%$ to $10.4 \%$ ), chronic kidney disease (from $3.9 \%$ to $5.0 \%$ ), and heart failure (from $7.3 \%$ to $9.3 \%$ ) also increased during the study periods. The frequencies of brain CT use was decreased from $94.4 \%$ in 2000 2003 to $79.6 \%$ in 2008-2011 while brain MRI use was increased from $22.1 \%$ in $2000-2003$ to $55.4 \%$ in $2008-2011$. General characteristics are described in Table 1. During the study periods, 30-day in-hospital mortality was from $1.7 \%$ to $1.9 \%$.

About 37\% of first-ever ischemic stroke patients received calcium channel blockers for antihypertension therapy during admission throughout the study period. The use of angiotensin receptor blockers was increased from $6 \%$ to $18.1 \%$ during the study periods. Frequencies of angiotensin-converting-enzyme inhibitors $(16 \%)$ and diuretics $(13 \%)$ were much the same among three time sections. The use of statin in ischemic stroke patients was increased from $8.8 \%$ in $2000-2003,21.9 \%$ in 2004-2007, and to $30.8 \%$ in 2008-2011. Stroke patients received antiplatelet were $76.1 \%$ in $2000-2003$, $85.3 \%$ in 2004 2007 and $86.6 \%$ in 2008-2011, respectively. There was $30.0 \%$ in 2000-2003, 28.9\% in 2004-2007, and 34.5\% in 2008-2011, respectively, of ischemic stroke patients with atrial fibrillation received oral anticoagulant agents during the hospitalization.

The incidence rate of ischemic stroke for people aged 18 years or older decreased gradually from 2000 (142.3 per 
Table 1. Characteristic of hospitalized first-ever ischemic stroke patients from Taiwanese National Health Insurance Database $(2000-2011), \mathrm{N}=291,381$

\begin{tabular}{|c|c|c|c|c|}
\hline & $\begin{array}{c}\text { Year 2000-2003 } \\
\quad N=92,525\end{array}$ & $\begin{array}{c}\text { Year 2004-2007 } \\
\quad \mathrm{N}=95,681\end{array}$ & $\begin{array}{c}\text { Year 2008-2011, } \\
\quad N=94,543\end{array}$ & $P$ value \\
\hline Age (median, interquartile range [IQR]) & $70(61-77)$ & $69(59-77)$ & $69(59-78)$ & 0.0819 \\
\hline \multicolumn{5}{|l|}{ Sex } \\
\hline Male (\%) & 57.2 & 58.9 & 59.5 & $<0.0001$ \\
\hline Length of stay (day) (median, IOR) & $7(5-13)$ & $7(5-14)$ & $7(5-14)$ & 0.0939 \\
\hline Computed tomography (CT) (\%) & 94.4 & 85.1 & 79.6 & $<0.0001$ \\
\hline Magnetic resonance imaging (MRI) (\%) & 22.8 & 38.0 & 55.0 & $<0.0001$ \\
\hline \multicolumn{5}{|l|}{ Medical history (\%) } \\
\hline Hypertension & 58.9 & 63.4 & 62.5 & $<0.0001$ \\
\hline Diabetes & 29.7 & 33.1 & 34.0 & $<0.0001$ \\
\hline Hyperlipidemia & 22.0 & 34.1 & 40.7 & $<0.0001$ \\
\hline Ischemic Heart Disease & 24.2 & 28.9 & 28.5 & $<0.0001$ \\
\hline Atrial Fibrillation & 7.3 & 8.9 & 10.3 & $<0.0001$ \\
\hline Chronic Kidney Disease & 4.0 & 4.5 & 5.0 & $<0.0001$ \\
\hline Heart Failure & 7.3 & 8.8 & 9.4 & $<0.0001$ \\
\hline 30 day in-hospital mortality (\%) & 1.7 & 1.6 & 1.9 & $<0.0001$ \\
\hline \multicolumn{5}{|l|}{ Medications } \\
\hline Calcium channel blockers (CCBs) & 36.6 & 36.4 & 36.3 & 0.2651 \\
\hline Angiotensin-converting-enzyme inhibitors (ACEls) & 15.2 & 18.0 & 15.1 & 0.826 \\
\hline Angiotensin receptor blockers (ARBs) & 6.2 & 14.7 & 18.6 & $<0.0001$ \\
\hline Diuretics & 11.0 & 13.5 & 12.6 & $<0.0001$ \\
\hline Statins & 8.7 & 21.7 & 30.7 & $<0.0001$ \\
\hline Antiplatelet agents & 75.7 & 85.1 & 86.3 & $<0.0001$ \\
\hline Warfarin or novel oral anticoagulants (NOACs) for atrial fibrillation & 29.8 & 28.8 & 34.2 & $<0.0001$ \\
\hline
\end{tabular}

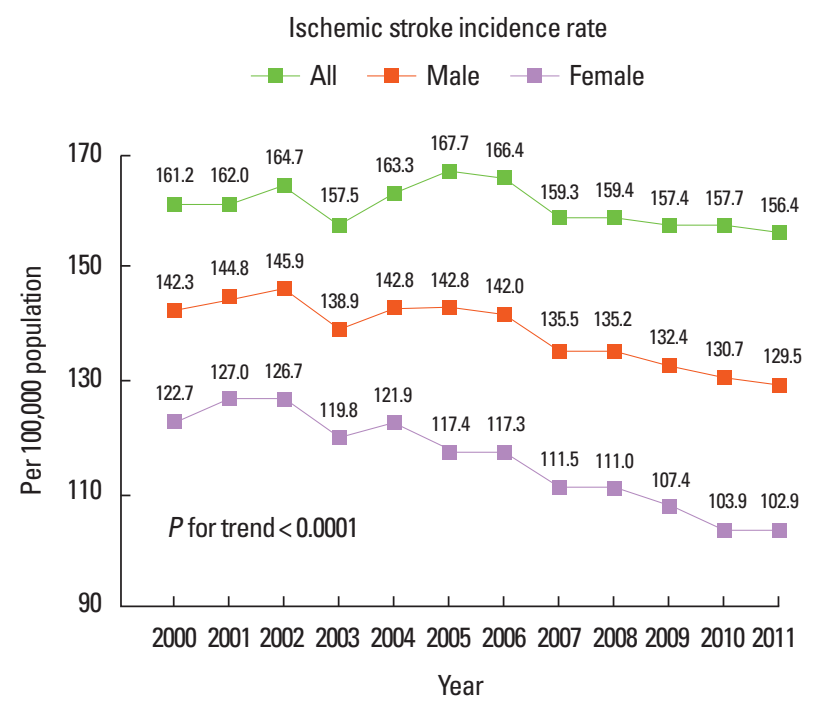

Figure 1. Trend of stroke incidence rate. Source: Taiwan National Health Insurance Database and Ministry of Interior.

100,000 persons) to 2011 ( 129.5 per 100,000 persons) except there was an obvious drop in 2003 then increased in 2004 (Figure 1). One-year stroke recurrent rate decreased gradually which was $9.61 \%$ in 2000 and decreased to $7.27 \%$ in 2011 except there was an obvious drop in 2003 then increased in 2004 (Figure 2). Compare to women, men had a higher incident rate and recurrent rate of stroke. We suspected the Severe Acute Respiratory Syndrome, 14 March 2003 through 5 July 2003 in

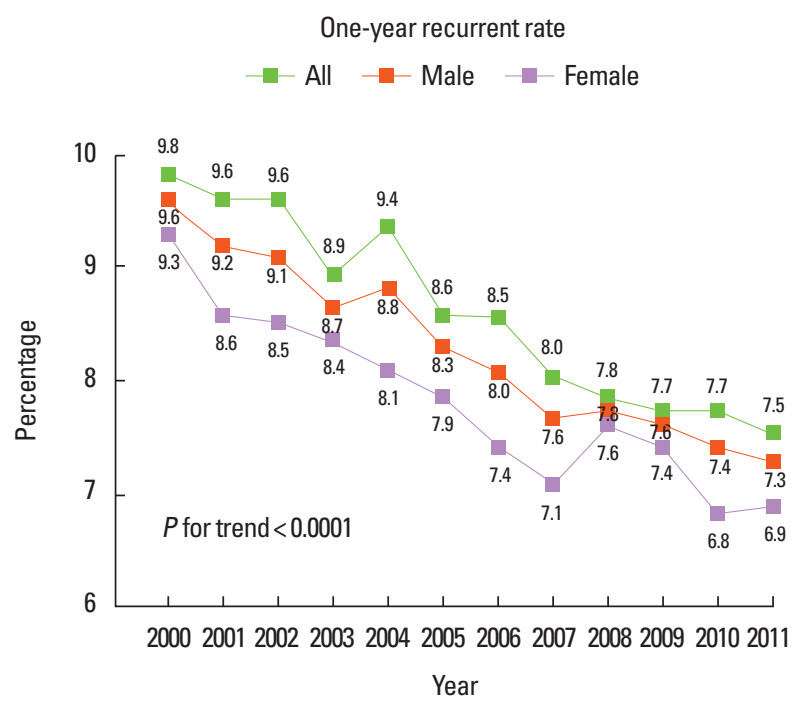

Figure 2. Trend of one-year stroke recurrent rate.

Taiwan, may cause decreasing rate of ischemic stroke hospitalization.

To explore the possible explanation for the drop of stroke incidence in 2003, we compared the stroke incidence in March through July from 2002 to 2004 and found that stroke incidence was substantially decreased in May and June, 2003, as compared to May and June in 2002 and 2004 (Table 2). 
Table 2. Crude stroke incidence rate from March to July between 2002 and 2004 (per 100,000 population)

\begin{tabular}{lrrrrr}
\hline \multirow{2}{*}{ Year } & \multicolumn{5}{c}{ Month } \\
\cline { 2 - 6 } & March & April & May & June & July \\
\hline 2002 & 12.94 & 12.96 & 12.52 & 11.70 & 12.53 \\
2003 & 12.83 & 11.54 & 9.27 & 9.91 & 12.19 \\
2004 & 12.91 & 11.54 & 12.13 & 12.36 & 11.81 \\
\hline
\end{tabular}

\section{Discussion}

This study provided an overview of baseline characteristics, risk factors, medication use during hospitalization, incidence, and one-year recurrence of first-ever ischemic stroke using the entire population of Taiwan from 2000 to 2011. Several findings, strengths and limitations merit discussion.

We used stroke hospitalization to represent incidence and recurrence of ischemic stroke with the assumption that all acute ischemic stroke patients should be hospitalized and the current health care system in Taiwan supports and encourages such a practice. However, a very small proportion of patients, likely those with minor stroke, may receive brain imaging at emergency departments or outpatient clinics but not be hospitalized due to various reasons, such as non-availability of beds or patients not wanting to wait. We found both incidence and recurrence of stroke dropped in May and June in 2003, which was during the fierce SARS epidemic in Taiwan. During that period of time, patients and medical doctors were less inclined to facilitate hospitalization unless the patient's condition was highly severe. Thus many patients with strokes of relatively minor severity may not have been counted in our analysis thereby potentially underestimating the real incidence and recurrence of ischemic stroke during that period of time.

Two studies exploring the incidence of stroke were conducted in $1980 \mathrm{~s}^{13}$ and $1990 \mathrm{~s},{ }^{14}$ respectively, in certain communities in Taiwan. The annual incidence rate of stroke for people aged 36 years or older in a study, from 1986 to 1990 , was 330 per 100,000 population. ${ }^{13}$ The other population-based stroke survey from 1993 to1996 reported that the annual incidence rate of stroke for people aged 50 years or older was 527 per 100,000 population. ${ }^{14}$ Since the current population was age 18 or older and we focused on incidence of ischemic stroke, as well as incidence of stroke was likely declining from 1986 to 2011, the annual incidence rate was lower in this study.

The characteristics of the stroke risk factors in this study is generally consistent with a large stroke registry from 2006 to 2008 but the rates of statin (30\% vs. 38\%) and antiplatelet ( $87 \%$ vs. $94 \%$ ) use during hospitalization were somewhat lower in this study. ${ }^{15}$ While the current study included only first- ever ischemic stroke, the prior study ${ }^{15}$ also included $34 \%$ recurrent ischemic stroke patients, which may have led the treating medical doctors to adopt a more aggressive vascular risk reduction strategy. Also hospitals participating in a stroke registry are presumably highly motivated to improve care quality and may generally have more awareness of/interest in the use of evidence-based therapies for stroke risk reduction. We found mortality rate was lower in this study, as compared to the prior stroke registry study $\left(1.9 \%\right.$ vs. $\left.4 \%{ }^{15}\right)$ which is likely due to the fact that this study only enrolled first-ever ischemic stroke.

It is informative to compare the trends in stroke incidence, recurrence, and management of stroke in Taiwan with data from other Asian countries, such as Korea. Among hospital admissions with stroke as the primary diagnostic code during 2001 to 2009, hospitalizations for ischemic stroke increased substantially in Korea. ${ }^{16}$ This apparent rise in annual ischemic stroke incidence in Korea was likely due to an increase in the country's elderly population. ${ }^{16}$ However, the median age of the current study in Taiwan did not change during the study period. Moreover, a comparison of survey data obtained in 2002 vs. 19931996 showed a significant improvement in hypertension awareness, treatment, and control in Taiwan, which may have contributed to its declining incidence of ischemic stroke. ${ }^{17}$ Ischemic stroke incidence in the population aged 35-74 years was 226 per 100,000 person-years (including first-ever and recurrent ischemic stroke) in Korea 146 per 100,000 persons in our population aged 18 years or older (first-ever ischemic stroke only); while the annual rate of recurrent ischemic stroke was about $5.0 \%$ in Japan during 2007 to 2009 vs. a $7.7 \%$ annual recurrent stroke (ischemic or hemorrhagic) rate noted in Taiwan. ${ }^{18}$

In the Fukuoka ischemic stroke registry, mean age was 70 years, $76 \%$ had hypertension, $27 \%$ diabetes mellitus, $46 \%$ hyperlipidemia, and $26 \%$ atrial fibrillation. ${ }^{19}$ Our study had similar frequencies of stroke risk factors except only $10 \%$ patients had atrial fibrillation in this study. Since diagnosis of atrial fibrillation in hospitalized ischemic stroke patients typically relies on a single baseline 12-lead electrocardiogram or history of atrial fibrillation, it is conceivable that the frequency of paroxysmal atrial fibrillation in admitted ischemic stroke patients in Taiwan might be underestimated. Among ischemic stroke patients with atrial fibrillation in this study, $34 \%$ vs. $10 \%$ in a Chinese ischemic stroke registry, ${ }^{20}$ received oral anticoagulants during hospitalization. The underuse of oral anticoagulant therapy in eligible Asian stroke patients during hospitalization may in part be due to concerns about hemorrhagic transformation. Also, 31\% patients received statin therapy in this study at discharge vs. 35\% in Fukuoka stroke registry, which is highly comparable. ${ }^{21}$ 
As compared to a prior study based on the control arms of clinical trials for secondary stroke prevention, the current study had higher recurrent rate of stroke $\left(8 \%\right.$ vs. $\left.5 \%{ }^{10}\right)$. The difference may exist in the prior study excluded patients with atrial fibrillation or severe carotid stenosis ${ }^{10}$ while this study included all first-ever ischemic stroke patients. Also, people participating in clinical trials may receive more rigorous risk factors control, which turns out to have lower recurrent stroke. Furthermore, the current study enrolled patients in the acute stage when the risk of recurrent stroke is high, whereas secondary stroke prevention trials, in general, enrolled patients after the acute stage. We found the one-year recurrent stroke was declining from 2000 to 2011, which may be explained by increasing use of statin (8.8\% to $30.8 \%$ ), antiplatelet agents ( $76.1 \%$ to $86.6 \%)$, and oral anticoagulant agents in atrial fibrillation (30.0\% to $34.5 \%)$ during hospitalization among this period of time.

In addition to the limitations mentioned above, NHIRD does not provide information on a few established stroke risk factors, e.g. smoking and blood pressure levels during followup period. Also, we did not explore medication use at outpatient clinics after discharge from the first-ever ischemic stroke. Thus we could not explore the impact of these factors on the incidence and recurrence of stroke.

In conclusion, in accord with recent trends around the world, ${ }^{2-5}$ we observed that the annual incidence and one-year recurrence rates of stroke declined over the last decade in Taiwan. However, it would seem that the magnitude of reduction for incident stroke seen in Western nations of approximately $20 \%$ was much greater than the more modest $9 \%$ decrease noted in Taiwan. We observed a rise in the use of statins, antiplatelet agents, and oral anticoagulant agents for atrial fibrillation in Taiwan during this period of time, which probably contributed to the declining rate of stroke, but there appears to more room for improvement in risk factor control if Taiwan hopes to gain at least the same level of decrease in incident stroke observed in Western countries.

\section{References}

1. Granger BB, Swedberg K, Ekman I, Granger CB, Olofsson B, McMurray JJ, et al. Adherence to candesartan and placebo and outcomes in chronic heart failure in the CHARM programme: double-blind, randomised, controlled clinical trial. Lancet 2005; 366:2005-2011.

2. Hall MJ, Levant S, DeFrances CJ. Hospitalization for stroke in US hospitals, 1989-2009. NCHS Data Brief 2012:1-8.

3. Rosengren A, Giang KW, Lappas G, Jern C, Torén K, Björck L.
Twenty-four-year trends in the incidence of ischemic stroke in Sweden from 1987 to 2010. Stroke 2013;44:2388-2393.

4. Wang Y, Rudd AG, Wolfe CD. Trends and survival between ethnic groups after stroke: the South London Stroke Register. Stroke 2013;44:380-387.

5. Ovbiagele B. National sex-specific trends in hospital-based stroke rates. J Stroke Cerebrovasc Dis 2011;20:537-540.

6. Gu Q, Burt VL, Dillon CF, Yoon S. Trends in antihypertensive medication use and blood pressure control among United States adults with hypertension: the National Health and Nutrition Examination Survey, 2001 to 2010. Circulation 2012; 126:2105-2114.

7. Shih CL, Chen CH, Sheu CF, Lang HC, Hsieh CL. Validating and improving the reliability of the EORTC qlq-c30 using a multidimensional Rasch model. Value Health 2013;16:848-854.

8. Tsai CT, Lai LP, Hwang JJ, Chen WP, Chiang FT, Hsu KL, et al. Renin-angiotensin system component expression in the HL-1 atrial cell line and in a pig model of atrial fibrillation. $J$ Hypertens 2008;26:570-582.

9. Lee M, Wu YL, Saver JL, Lee HC, Lee JD, Chang KC, et al. Is clopidogrel better than aspirin following breakthrough strokes while on aspirin? A retrospective cohort study. BMJ Open 2014; 4:e006672.

10. Hong KS, Yegiaian S, Lee M, Lee J, Saver JL. Declining stroke and vascular event recurrence rates in secondary prevention trials over the past 50 years and consequences for current trial design. Circulation 2011;123:2111-2119.

11. Hsieh CY, Chen CH, Li CY, Lai ML. Validating the diagnosis of acute ischemic stroke in a national health insurance claims database. J Formos Med Assoc 2015;114:254-259.

12. Cheng CL, Kao YH, Lin SJ, Lee CH, Lai ML. Validation of the National Health Insurance Research Database with ischemic stroke cases in Taiwan. Pharmacoepidemiol Drug Saf 2011;20: 236-242.

13. Hu HH, Sheng WY, Chu FL, Lan CF, Chiang BN. Incidence of stroke in Taiwan. Stroke 1992;23:1237-1241.

14. Fuh JL, Wang SJ, Liu HC, Shyu HY. Incidence of stroke on Kinmen, Taiwan. Neuroepidemiology 2000;19:258-264.

15. Hsieh FI, Lien LM, Chen ST, Bai CH, Sun MC, Tseng HP, et al. Get With the Guidelines-Stroke performance indicators: surveillance of stroke care in the Taiwan Stroke Registry: Get With the Guidelines-Stroke in Taiwan. Circulation 2010;122:1116-1123.

16. Hong KS, Bang OY, Kang DW, Yu KH, Bae HJ, Lee JS, et al. Stroke statistics in Korea: part I. Epidemiology and risk factors: a report from the Korean stroke society and clinical research center for stroke. J Stroke 2013;15:2-20.

17. Su TC, Bai CH, Chang HY, You SL, Chien KL, Chen MF, et al. Evidence for improved control of hypertension in Taiwan: 
1993-2002. J Hypertens 2008;26:600-606.

18. Suzuki N, Sato M, Houkin K, Terayama Y, Uchiyama S, Daida $\mathrm{H}$, et al. One-year atherothrombotic vascular events rates in outpatients with recent non-cardioembolic ischemic stroke: the EVEREST (Effective Vascular Event REduction after STroke) registry. J Stroke Cerebrovasc Dis 2012;21:245-253.

19. Ishitsuka K, Kamouchi M, Hata J, Fukuda K, Matsuo R, Kuro$\mathrm{da}$ J, et al. High blood pressure after acute ischemic stroke is associated with poor clinical outcomes: Fukuoka Stroke Regis- try. Hypertension 2014;63:54-60.

20. Wang C, Yang Z, Wang C, Wang Y, Zhao X, Liu L, et al. Significant underuse of warfarin in patients with nonvalvular atrial fibrillation: results from the China national stroke registry. $J$ Stroke Cerebrovasc Dis 2014;23:1157-1163.

21. Makihara N, Kamouchi M, Hata J, Matsuo R, Ago T, Kuroda J, et al. Statins and the risks of stroke recurrence and death after ischemic stroke: the Fukuoka Stroke Registry. Atherosclerosis 2013;231:211-215. 